\title{
ORIGINAL RESEARCH \\ Metal Artifact Reduction for Clipping and Coiling in Interventional C-Arm CT
}

D. Prell

Y. Kyriakou

T. Struffert

A. Dörfler

W.A. Kalender
BACKGROUND AND PURPOSE: Metallic implants induce massive artifacts in CT images which deteriorate image quality and often superimpose structures of interest. The purpose of this study was to apply and evaluate a dedicated MAR method for neuroradiologic intracranial clips and detachable platinum coiling events. We here report the first clinical results for MAR in FDCT.

MATERIALS AND METHODS: FDCT volume scans of several patients treated with endovascular coiling or intracranial clipping were corrected by using a dedicated FDCT MAR correction algorithm combined with an edge-preserving attenuation-normalization method in the projection space. Corrected and uncorrected images were compared by 2 experienced radiologists and evaluated for several imagequality features.

RESULTS: After application of our algorithm, implant delineation and visibility were highly improved. CT values compared with values in metal artifact-unaffected areas showed good agreement (average correction of $1300 \mathrm{HU}$ ). Image noise was reduced overall by $27 \%$. Intracranial hemorrhage in the direct surroundings of the implanted coil or clip material was displayed without worrisome metal artifacts, and our algorithm even allowed diagnosis in areas where extensive information losses were seen. The high spatial resolution provided by FDCT imaging was well preserved.

CONCLUSIONS: Our MAR method provided metal artifact-reduced images in every studied case. It reduced image noise and corrected $\mathrm{CT}$ values to levels comparable with images measured without metallic implants. An overall improvement of brain tissue modeling and implant visibility was achieved. MAR in neuroradiologic FDCT imaging is a promising step forward for better image quality and diagnosis in the presence of metallic implants.

ABBREVIATIONS: COR $=$ center of rotation; FD = flat panel detector; FDCT $=$ flat panel detector $\mathrm{CT} ; \mathrm{LI}=$ linear interpolation; $\mathrm{MAR}=$ metal artifact reduction; $\max =$ maximum; $\mathrm{ROI}=$ region of interest
D iagnostic and interventional neuroradiologic applications have profited from the availability of innovative developments such as FDs in rotational C-arm-based FDCT. ${ }^{1-6}$ Applications of FDCT range from imaging of intracranial hemorrhage to neurovascular imaging and interventions (ie, intracranial aneurysms and arteriovenous malformations) to spinal imaging and interventions. ${ }^{1,6-9}$ Especially since the International Subarachnoid Aneurysm Trial ${ }^{5,10}$ and the establishment of endovascular coiling as the treatment of choice for intracranial aneurysms, FDCT became a novel alternative to postinterventional CT. ${ }^{5,11-13}$ It offers not only higher spatial resolution in volumetric imaging compared with conventional CT but also rapid visualization of periprocedural hemorrhage and, therefore, improved rapid complication management without the need for patient transfer. ${ }^{13}$ This is necessary because management within 30 minutes will improve the patient's outcome. ${ }^{14}$ Nevertheless, artifacts induced by metallic objects, (ie, clips and detachable coils) reduce image quality and may prevent proper diagnosis of areas close to

Received June 19, 2009; accepted after revision August 7.

From the Institute of Medical Physics (D.P., Y.K., W.A.K.) and Department of Neuroradiology (T.S., A.D.), University of Erlangen-Nürnberg, Erlangen, Germany.

This work was supported by orthoMIT-Minimal Invasive Orthopedic Therapy, grant 01E00426, German Federal Ministry of Education and Research, Berlin, Germany.

Please address correspondence to Daniel Prell, Dipl Phys, Institute of Medical Physics, University of Erlangen-Nürnberg, Henkestra 91, 91052 Erlangen, Germany; e-mail: daniel. prell@imp.uni-erlangen.de

Indicates open access to non-subscribers at www.ajnr.org

DOI 10.3174/ajnr.A1883 the implants, (ie, recognition and management of complications such as rebleedings or the rupture of an aneurysm during endovascular treatment). ${ }^{5}$

Although many attempts for MAR in conventional CT were made, most algorithms did not provide sufficient quality to be accepted for clinical application. ${ }^{15-17}$ Also, the published correction algorithms were based on simple sinogram manipulations (ie, 1D linear interpolation of metal traces) ${ }^{18}$ and, therefore, were not compatible with modern FDs, which use Feldkamp-type reconstruction. ${ }^{19}$ A novel approach recently presented by Prell et $\mathrm{al}^{20}$ tackles the problem of metal artifacts by using an interpolation-based 3D correction algorithm in the raw data. We present a novel approach for reducing metalinduced artifacts in neuroradiologic applications, focusing on artifacts caused by clips and coils, especially designed for FD datasets. The corrections were applied to neuroradiologic CT images for improvements in implant and brain-tissue modeling to achieve an overall image quality improvement.

\section{Materials and Methods}

\section{Equipment}

All investigations were performed by using an Axiom Artis dBA Carm system (Siemens Healthcare Sector, Forchheim, Germany) for aquisition. The system is equipped with biplane imaging components, from which only 1 was used for CT imaging. The FD has an active area of $40 \times 30 \mathrm{~cm}^{2}$ (pixel size of $308 \mu \mathrm{m}$ at $2 \times 2$ pixel binning, $\mathrm{CsI}[\mathrm{Tl}] \mathrm{a}: \mathrm{Si})$. The system geometry allows a reconstruction of a nontruncated volume of approximately $22 \mathrm{~cm}$ (in-plane) and $16 \mathrm{~cm}$ (in the $\mathrm{z}$-direction). Figure $1 A,-B$ illustrates the system, its geometry, and 


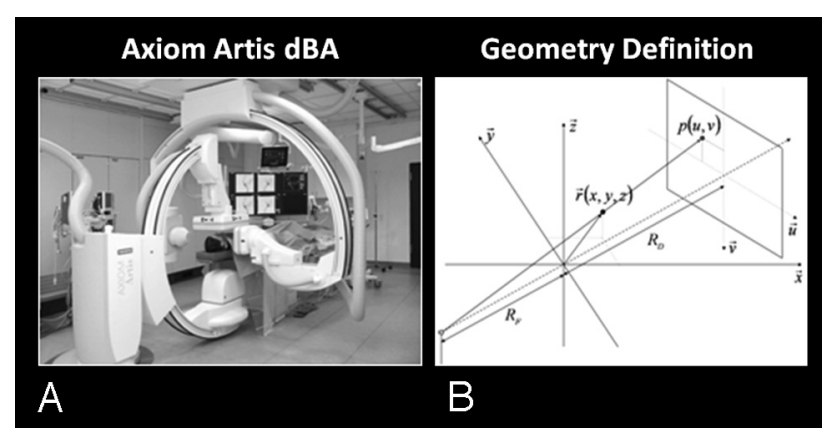

Fig 1. Photograph of the system in use (Axiom Artis dBA, Siemens Healthcare Sector) and a diagram of its geometry. RF and RD correspond to the distance from the focal spot to the COR and from the detector to the COR respectively. $R(x, y, z)$ defines the position in the $x / y$ and $x / z$-plane, and $p(u, v)$, the corresponding attenuation value measured by the respective detector pixel.

the reconstructable FOV. All acquisitions were performed by using the embedded high-quality DynaCT scan (Siemens, Erlangen, Germany) protocols with an aquisition range of $200^{\circ}$ (20 seconds for acquisition, $1240 \times 960$ projection matrix, $0.4^{\circ}$ angular increment) and 496 projections. The detector entrance-dose setting was routinely a value of $1.2 \mu \mathrm{Gy} /$ projection. The respective raw data were reconstructed with the dedicated reconstruction software ImpactFD (VAMP, Erlangen, Germany), by using a smooth kernel typical for the evaluation of bleeding.

\section{MAR Algorithm}

The MAR algorithm used here was based on the correction scheme described in Prell et al. ${ }^{20}$ We extended this correction method by combining it with an edge-preserving attenuation-normalization procedure in the raw data domain. ${ }^{21,22}$ The principle behind the proposed method is to start with a first reconstruction of the acquired raw data and then subsequently improve the MAR. In a first step, metal artifacts are largely reduced by interpolating the metal traces in the raw data domain, followed by a second Feldkamp reconstruction. ${ }^{19}$ The second correction step uses the attenuation-normalization procedure to include edge information, which is generally lost after interpolation. In a final combination procedure, the metal implants are reinserted into the corrected image. The quasi-iterative correction scheme is shown step-by-step in Fig 2.

\section{Metal Detection and First Correction Step}

The metal detection in the initially reconstructed image is performed by first smoothing the image by a gaussian filter ${ }^{20,23}$ to minimize the effect of mistakenly detecting artifacts as implant geometry. A perspective forward projection of the obtained subvolumes efficiently detects the metal implants in the raw data. A complete detection in the raw data without reconstructing an initial image has been shown to yield unsatisfactory results. ${ }^{18,20}$ The 3D LI correction algorithm uses the $3 \mathrm{D}$ raw data space to calculate surrogate data for the detected metal traces. The efficiency of detecting the metal implants in the raw data strongly depends on the accuracy of the geometry calibration of the system used for the forward projection and the size of the initial reconstruction. To avoid an insufficient interpolation of the metal traces due to a too small reconstructed initial image or misalignment in the geometry calibration of the system, we used an interpolation width to set the starting and ending points of the interpolation. For our method, a width of $\Delta n=3$ detector pixels was chosen. This results in a maximal transition area in the corrected image of approximately $0.6 \mathrm{~mm}$ from the implant to the unchanged tissue according to
1)

$$
\Delta r_{\text {transition }}=\frac{R_{\mathrm{F}}}{R_{\mathrm{FD}}} \cdot \Delta n,
$$

where $R_{F}$ is the distance from the COR to the focus and $R_{F D}$ is the distance from the focus to the detector (Fig $1 B$ ). Only CT values and resolution in the transition area are subject to noticeable influences by the interpolation scheme and, therefore, have to be considered separately.

The extensive spreading of metal artifacts in the reconstructed image is mainly caused by the Feldkamp reconstruction, which nonlinearily amplifies beam-hardening artifacts, scattered radiation, and photon starvation. By pragmatically interpolating the contaminated metal traces and thus replacing them with surrogate attenuation values, using attenuation values surrounding the metal traces and linearily combining them, these artifacts are reduced in the reconstructed image.

Subsequent reconstruction of the modified raw data yields an artifacts-reduced image without metallic objects. A final combination procedure reinserts these missing objects into the artifacts-reduced image to get the final corrected image.

\section{Second Correction Step}

One drawback of every interpolation scheme is the introduction of secondary artifacts. Although the proposed algorithm already showed an improved reduction of the most severe artifacts, secondary artifacts connecting the implants and structures of high attenuation still appeared. ${ }^{20-23} \mathrm{We}$, therefore, combined, in a second step, an attenuation-normalization method as proposed by Müller and Buzug $^{21}$ and extended by Meyer et $\mathrm{al}^{22}$ with the first interpolation correction. This correction procedure uses a multilevel segmentation, which distinguishes not only air and soft tissue but also soft tissue and bone. The different tissue classes are represented by different values in the segmented image ( $\operatorname{air}=0$, soft tissue $=1$, bone $=2$ ). This image is forward-projected to normalize the attenuation values in the raw data and to preserve edge information lost after interpolation. Subsequently, 3D LI is performed to get the final corrected image, followed by a denormalization of the interpolated raw data. The reconstruction of a first 3D LI corrected image is necessary to minimize the influence of metal artifacts on the segmented image because they can easily be detected as bony structures.

\section{Evaluation of CT Values, Image Noise, and Spatial Resolution}

For investigation of CT-value consistency and image noise before and after correction, a subvolume of the original scan was used. After reconstruction of the coarse image, a dedicated bone-removal algorithm removed bone and implant and air structures so that an investigation of soft tissue, which also contains the obscuring artifacts, was enabled. A simple threshold-based segmentation would falsely detect artifacts as bony structures or air inclusions. Figure $3 A$ shows the image containing only the soft-tissue-equivalent areas, which are used for evaluation. The difference in the maximal CT value in the softtissue region and the difference in image noise $\sigma$ before and after correction were calculated according to

2)

$$
\begin{gathered}
\Delta \mathrm{CT}_{\max }=\mathrm{C}_{\max }^{\text {uncorrected }}-\mathrm{CT}_{\max }^{\text {corrected }}, \\
\Delta \sigma=\sigma^{\text {uncorrected }}-\sigma^{\text {corrected }}
\end{gathered}
$$

Standardized spatial resolution investigations (eg, bar pattern and modulation transfer functions) cannot be performed in clinical datasets. Therefore, only a visual investigation of spatial resolution was 


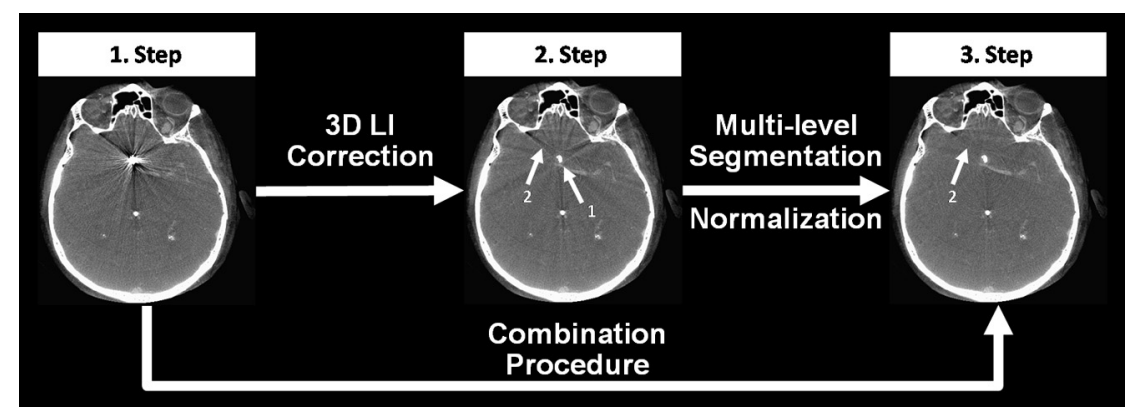

Fig 2. Diagram of the combination procedure. The first step corresponds to the initial reconstruction; the second step, to the first correction by using $3 \mathrm{D}$ LI; and the third step, to the attenuation-normalization correction. The combination procedure reinserts the metal implants into the corrected image. Arrow 1 shows a Guglielmi (Boston Scientific) detachable coil. Arrow 2 indicates metal artifacts and the subsequent reduction of these perturbations.

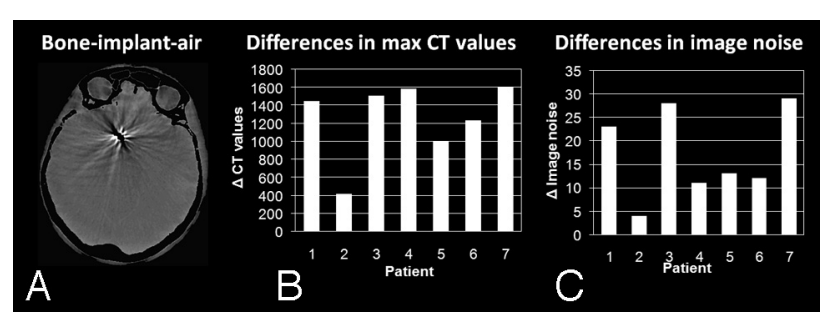

Fig 3. Investigated soft-tissue image $(A)$ and graphs of the results for the differences in CT values $(B)$ and image noise $(C)$.

performed. We, therefore, calculated difference images between the uncorrected and corrected image by using a sharp reconstruction kernel. General changes in spatial resolution would be indicated by visible object edges or other structural information besides the artifact structures. The aim of the MAR method was to reduce the metalinduced artifacts while not decreasing spatial resolution.

\section{Patient Cases}

A total of 7 consecutive rotational patient head scans were evaluated, in which 5 patients were treated with detachable platinum coil embolization; 1 patient, with detachable platinum coil embolization and additional clipping; and 1 patient only, with several intracranial clips. One patient showed subarachnoid hemorrhage, and 1 patient, intracerebral hemorrhage. The FDCT scan was performed at the end of the coiling procedure to rule out rebleeding and acute hydrocephalus and to confirm the correct positioning of an external ventricular drainage. We chose patients with a $>25-\mathrm{cm}$ coil length implanted, because beam-hardening artifacts would be most prominent in these patients. For the patients with clipping, the FDCT scanning was performed at the end of the control angiography after surgery to rule out hydrocephalus and to confirm the correct positioning of an external drainage.

All scans were carried out without additional contrast agent injection (uncollimated, full-field head scans). No special requirements were needed for this study because the raw data were processed only retrospectively. The images (uncorrected and corrected) were stored anonymized on a workstation for evaluation by 2 experienced neuroradiologists.

\section{Results}

\section{Evaluation of CT Values, Image Noise, and Spatial Resolution}

Results for the assessment of maximal CT value are shown in Fig $3 B$, where the differences between the maximal achieved
CT values in soft-tissue areas are listed. After we applied the correction algorithm, the maximal CT value, artificially changed by beam-hardening and metal-induced noise artifacts, was reduced by up to $1600 \mathrm{HU}$. Maximal CT values in the detected soft-tissue areas were reduced to a mean maximal value of $40 \mathrm{HU}$ after correction. On average, a reduction of $1300 \mathrm{HU}$ was achieved. MAR also yielded a more homogeneous CT-value distribution in soft-tissue and bony regions.

Image-noise evaluation (Fig $3 C$ ) shows a drastic reduction of metal-induced noise after correction. For all patients, image noise was reduced by typically $27 \%$, corresponding to a mean reduction of $17 \mathrm{HU}$. The correction achieved a lower image noise in every studied case, which also led to better lowcontrast detectability ${ }^{24}$ in these areas. The decrease of image noise in soft-tissue regions can be regarded as an indicator for an increase of homogeneity as shown by Fahrig et $\mathrm{al}^{24}$ and Prell et al. ${ }^{25}$

To evaluate the influence of the correction method on the spatial resolution of the corrected CT images, we used difference images to show explicitly which image areas were affected by our MAR method. Figure $4 A$ shows the uncorrected, the corrected, and the corresponding difference image (reconstructed by using a sharp reconstruction kernel to get a more precise indicator for spatial resolution changes) for patients 1 , 6 , and 7. As can clearly be seen in Fig $4 C,-F$, only metalinduced noise, beam-hardening, and scatter artifacts are corrected; no structural information other than the streak and noise artifacts is visible in the difference image. The correction process only changed image details related to metal artifacts. However, even in a narrow windowing, this additional information is not present, which confirms that the correction process preserves the spatial resolution provided by FDCT imaging.

\section{Implant Visibility, Brain-Tissue Imaging, and Overall Image Quality}

Figure 5 shows the uncorrected and corrected images for 3 patients in the sagittal view and in the sections corresponding to the dashed line in the subfigures of Fig $5 A,-D$, and $-\mathrm{G}$. Beam-hardening artifacts and photon starvation caused a drastic reduction of the CT values between the connecting lines of the coil and clips and reduced the CT values in these areas below the representable value of $-1024 \mathrm{HU}$. No information could be obtained. After correction, the brain tissue surrounding the implants is displayed without disturbing metal artifacts. Also, the implants themselves are displayed 


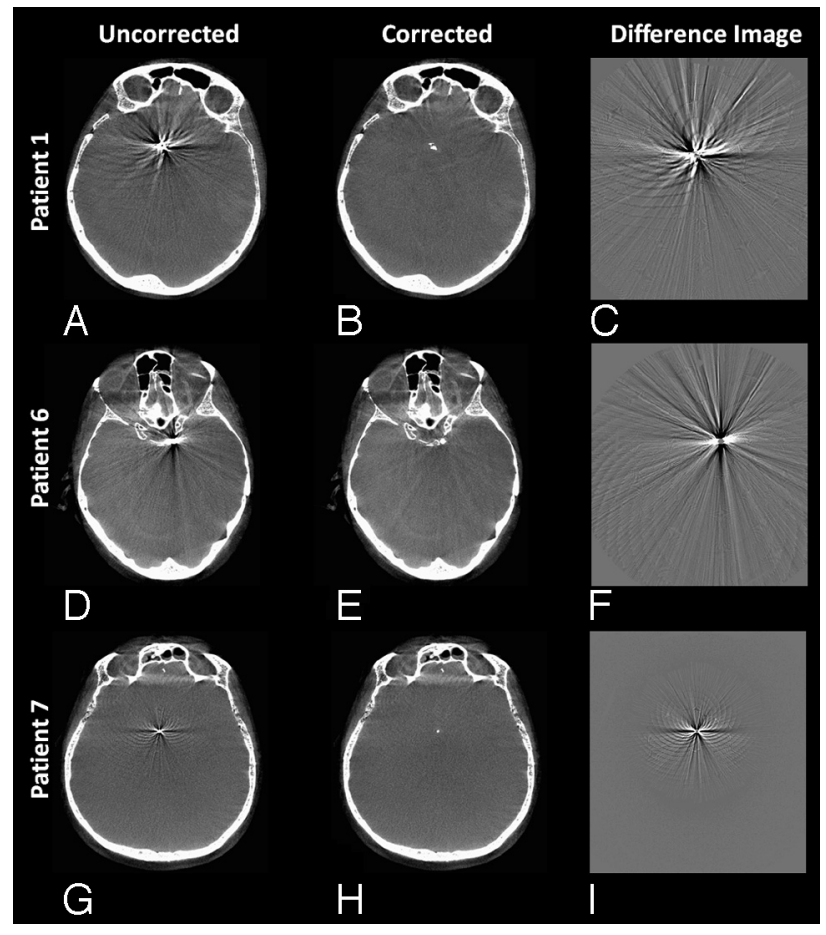

Fig 4. $A, D$, and $G$, Uncorrected images. $B, E$, and $H$, MAR-corrected images (windows setting of CO/W1000). C, F, and $I$, Corresponding difference images (narrower windows setting of CO/W500). The difference images apparently contain no other structural information than the metal-induced artifacts.
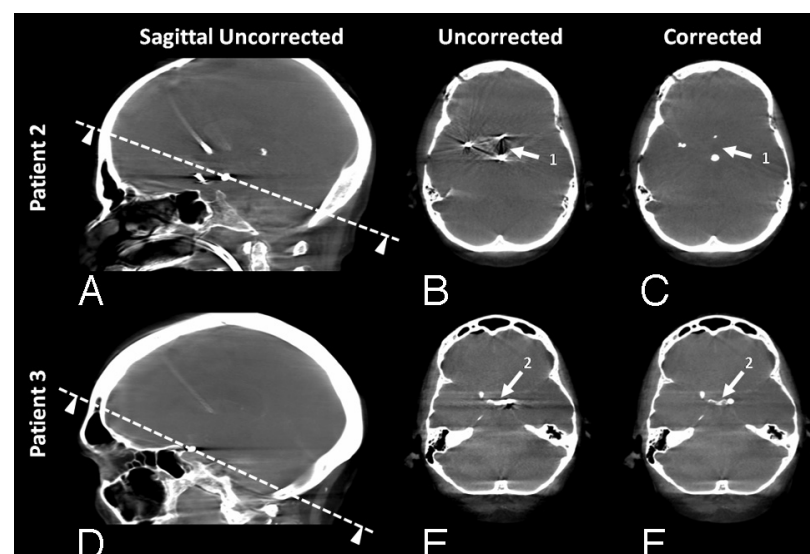

B

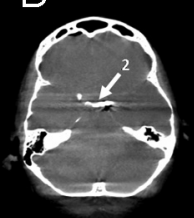

C

$\mathrm{D}$
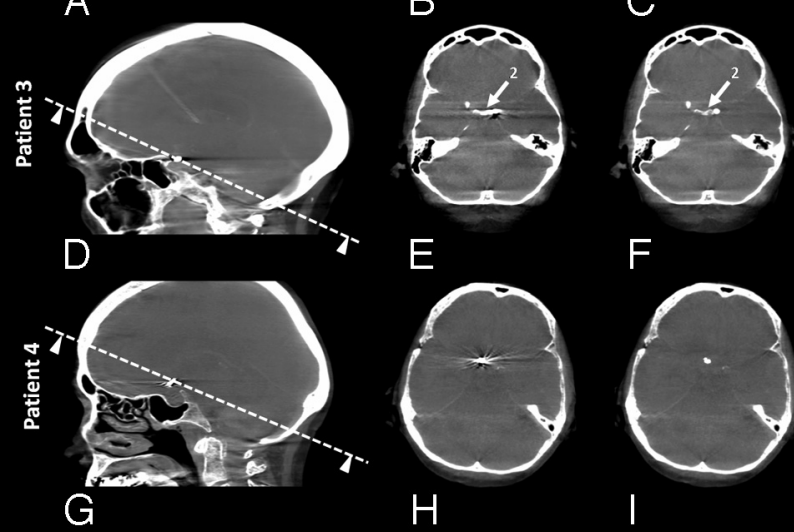

$\mathrm{F}$

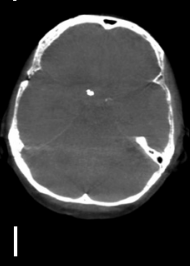

Fig 5. $A, D$, and $G$, Sagittal views of patients 2-4. $B, E$, and $H$, Uncorrected CT section corresponding to the dashed line. $C, F$, and $I$, Corrected CT section. Arrow 1 shows the restored $C T$ values and information between the clips and coils after correction. Arrow 2 indicates the bony structures visible after correction even close to the detechable coil (CO/W1000)

more clearly than before correction. Correction now provides information even for areas that were severely contaminated with artifacts as depicted by arrow 1 in Fig $5 C$. The clips and detachable coils used for embolization are displayed without beam-hardening or noise artifacts after correction. Also, fine

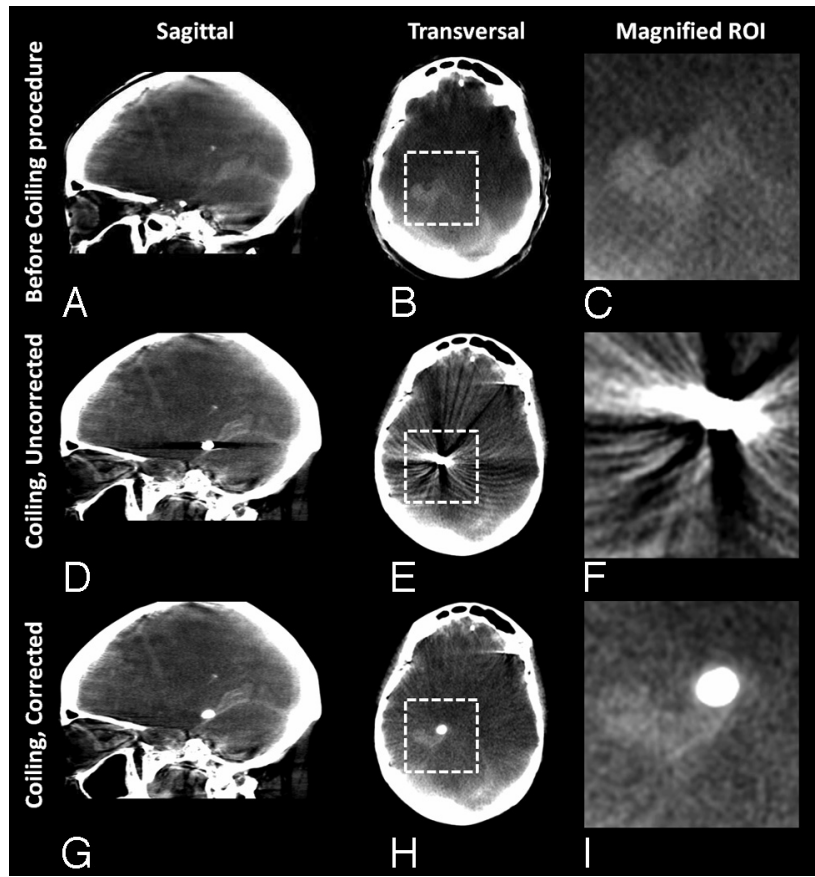

Fig 6. Scans of patient 5. $A-C$, Before coiling, sagittal image $(A)$, transversal image $(B)$, and the corresponding magnified $\mathrm{ROI}(C)$. D-F, After coiling, sagittal image $(D)$, uncorrected transversal image $(E)$; and the corresponding magnified ROI $(F)$. G-I, Sagittal corrected image $(G)$, corrected transversal image $(H)$, and the corresponding magnified ROI (I). After MAR is applied, one can recognize the size and configuration of the bleeding in the same way as before the coiling procedure (CO/W250).

bone structures previously superimposed by metal-induced artifacts become visible. Arrow 2 in Fig $5 E,-F$ shows bony structures after correction, which could have been mistakenly detected as metal artifacts in the original image. Imaging of the close vicinity (down to $0.58 \mathrm{~mm}$ from the implants) of the clips and coils is also improved; this change allows a proper diagnosis even of these areas.

This improvement becomes even more obvious by looking at Fig 6. Shown here is patient 5, treated with Guglielmi detachable coils (Boston Scientific, Natick, Massachusetts). The first FDCT, acquired before the intervention, demonstrates the hemorrhage caused by the aneurysm. Notice the configuration of the hemorrhage. The coiling procedure was uneventful. After successful endovascular therapy, the uncorrected images (Fig 6D-F) show massive artifacts caused by the coil package. In the axial images (Fig $6 E$, magnification in Fig $6 F$ ), the surroundings of the aneurysm are completely masked by artifacts. After MAR (Figs $6 G-I$ ), the artifacts are eliminated and the configuration of the hemorrhage can be recognized as it was before the coiling procedure. The coil package can be recognized as a bright oval structure. The disturbing artifacts are reduced to a large degree so that proper analysis of the implant, the surrounding tissue, and the bleeding itself is enabled. One drawback seems to be the imaging of the transition area between the implant and surrounding tissue. Although drastic improvement in imaging of these areas was achieved, due to the interpolation scheme in the raw data, these areas contain miscalculated CT values.

\section{Discussion}

FDCT is increasingly used after embolization of intracranial aneurysms, arteriovenous malformations, or intracranial 
stent placement to recognize potential hemorrhagic complications as soon as possible. In the presence of metallic implants, artifacts caused by the metal often obscure image details and may prevent a proper diagnosis of areas of interest. It is, therefore, of great importance to reduce these deteriorating artifacts to a minimum for better diagnosis. In this study, a new MAR approach was tested and evaluated. The MAR algorithm especially designed for FDCT systems and geometries provided better image quality with respect to the visualization of brain tissue surrounding the implants in every studied case.

Metal artifacts, mainly due to beam-hardening, scattered radiation, sampling, and noise artifacts were largely reduced after correction. This reduction not only achieved an overall improved image quality without the disturbing artifacts but also allowed a diagnosis of areas previously obscured by these artifacts. Especially during embolization by using detachable platinum coils, our algorithm proved to be a promising addition to standard FDCT imaging because better imaging of the implant and areas close to the implants was achieved. This becomes even more important when routine follow-up scanning has to be performed to investigate the position of the implant or to rule out rebleedings after embolization. Our approach improved the visibility of intracerebral hemorrhage close to metallic objects and may allow a more precise recognition of rebleeding during interventions because rebleeding may be masked by metal artifacts. One can assume that MAR may be useful in the visualization of other implants that need a high spatial resolution, like cochlear implants, stapes prostheses, or ventricular drainages.

One limitation is related to the imaging of the transition area to the metal implant. Although artifacts were largely reduced, resolution losses close to the implant can occur if the attenuation values in the raw data are not replaced by proper surrogate data; thus, reliable CT values of the transition area between implant and surrounding tissue can only be given with reserve. Incorrect CT values could lead to misinterpretations in these areas. However, this area of noticeable influence is here restricted to $0.6 \mathrm{~mm}$ from the implant. Before correction, the transition zone was not evaluable due to the massive metal artifacts. Nevertheless, by comparing the drawbacks and assets of our correction algorithm, one can see that an overall improvement of implant visibility and image quality was achieved. Another drawback may be that every raw data-based correction scheme entails a modification of raw data, and secondary artifacts introduced by the correction scheme cannot be excluded. The clinical evaluation of the images, therefore, has to include the uncorrected and the corrected version of the images. Although progress in terms of suppressing these secondary artifacts was made, negative effects still have to be monitored.

Interpolation-based MAR in FDCT shows promising results in these cases, which appear to be better than results shown in other publications regarding conventional CT. ${ }^{16,20,21,23}$ One reason for this is the smaller sampling distance (ie, spatial resolution provided by the FD). While conventional CT provides detector-element sizes of approximately $0.6 \mathrm{~mm}$, FDs achieve sizes down to $154 \mu \mathrm{m}$. This allows a much closer interpolation to the implants in the raw data. Another advantage of the method shown here seems to be the correction in the raw data itself (ie, on every perturbed detector pixel), whereas most published corrections in conventional CT were performed in the subsequent calculated sinogram after acquisition. ${ }^{16,18,21,23}$ Furthermore, our algorithm uses not only the attenuation values in each projection for calculating surrogate attenuation values but also the attenuation values from different projection angles, making the interpolation scheme truly 3D.

The algorithm itself can be easily included in a daily routine because no special requirements are needed for the FDCT system, and the correction and segmentation processes work automatically. Computational times compared with those in standard reconstruction can also be largely reduced by combining MAR with acceleration techniques on graphic processing units, ${ }^{26,27}$ which gives the radiologist the uncorrected and corrected image within almost the same time.

\section{Conclusions}

The MAR algorithm presented here provides an improved visibility in the close vicinity of the intracranial clips and endoaneurysmal coil packages in this limited sample of patient datasets. The obscuring metal artifacts were largely reduced, and an increase in homogeneity in soft-tissue regions was achieved. A better detectability of hemorrhage during or after endovascular embolization may be achieved. A large-scale clinical study is still pending.

\section{References}

1. Dörfler A, Struffert T, Engelhorn T, et al. Rotational flat-panel computed tomography in diagnostic and interventional neuroradiology. Rofo 2008; 180:891-98

2. Södermann M, Babic D, Holmin S, et al. Brain imaging with a detector C-arm: technique and clinical interest of XperCT. Neuroradiology 2008;50:863-68

3. Kalender WA, Kyriakou Y. Flat-detector computed tomography. Eur Radiol 2007; 17:2767-79

4. Doelken M, Struffert T, Richter G, et al. Flat-panel detector volumetric CT for visualization of subarachnoid hemorrhage and ventricles: preliminary results compared to conventional CT. Neuroradiology 2008;50:517-23. Epub 2008 Mar 11

5. Richter G, Engelhorn T, Struffert T, et al. Flat panel detector angiographic CT for stent-assisted coil embolization of broad-based cerebral aneurysms. AJNR Am J Neuroradiol 2007;28:1902-08

6. Kyriakou Y, Richter G, Dörfler A, et al. Neuroradiologic applications with routine $\mathrm{C}$-arm flat panel detector $\mathrm{CT}$ : evaluation of patient dose measurements. AJNR Am J Neuroradiol 2007;29:1930-36

7. Struffert T, Richter G, Engelhorn T, et al. Visualization of intracerebral hemorrhage with flat-detector CT compared to multislice CT: results in 44 cases. Eur Radiol 2009;19:619-25

8. Engelhorn T, Struffert T, Richter G, et al. Flat panel detector angiographic $\mathrm{CT}$ in the management of aneurysmal rupture during coil embolization. AJNR Am J Neuroradiol 2008;29:1581-84

9. Struffert T, Buhk JH, Buchfelder M, et al. Coil migration after endovascular coil occlusion of internal carotid artery pseudoaneurysms within the sphenoid sinus. Minim Invasive Neurosurg 2009;52:89-92. Epub 2009 May 18

10. Molyneux AJ, Kerr RS, Yu LM. International Subarachnoid Aneurysm Tria (ISAT) of neurosurgical clipping versus endovascular coiling in 2143 patients with ruptured intracranial aneurysms: a randomised comparison of effects on survival, dependency, seizures, rebleeding, subgroups and aneurysm occlusion. Lancet 2005;366:809-17

11. Wanke I, Gizewski E, Dörfler A, et al. Stenting plus coiling: dangerous or helpful [in German]? Rofo 2005;177:1255-59

12. Wanke I, Dörfler A, Göricke S, et al. Treatment of wide-necked intracranial aneurysms with a self-expanding stent: mid-term results. Zentralbl Neurochir 2005;66:163-69

13. Wanke I, Egelhof T, Dörfler A, et al. Intracranial aneurysms: pathogenesis, rupture risk, treatment options [in German]. Rofo 2003;175:1064-1070

14. Carvi Y, Nievas M, Haas E, et al. Severe intracranial bleedings during endovascular procedures: outcome of surgically treated patients. Neurol Res 2007;29:81-90

15. Yazdia M, Gingras L, Beaulieu L. An adaptive approach to metal artifact reduc- 
tion in helical computed tomography for radiation therapy treatment planning: experimental and clinical studies. Int J Radiat Oncol Biol Phys 2005;62:1224-31

16. Bal M, Spies L. Metal artifact reduction in CT using tissue-class modeling and adaptive prefiltering. Med Phys 2005;33:2852-59

17. Oehler M, Buzug TM. Statistical image reconstruction for inconsistent CT projection data. Methods Inf Med 2005;46:261-69

18. Kalender WA, Hebel R, Ebersberger J. Reduction of CT artifacts caused by metallic implants. Radiology 1987;164:576-77

19. Feldkamp LA, Davis LC, Kress JW. Practical cone-beam algorithm. J Opt Soc Am 1984;A1:612-19

20. Prell D, Kyriakou Y, Kalender WA. A dedicated raw data-based metal artifact reduction method for flat-detector CT. European Radiology 19, Suppl. 1:257 B-514, March 2009, Vienna, Austria

21. Müller J, Buzug TM. Spurious structures created by interpolation-based CT metal artifact reduction. Med Imaging 2009: Physics of Medical Imaging, Proc. SPIE, vol. 7258, 13; March 2009; Lake Buena Vista, Florida
22. Meyer E, Berger F, Raupach R, et al. Normalized Metal Artifact Reduction (NMAR) in computed tomography. In: IEEE Medical Imaging Conference Record. 2009. Proceedings M09-206, October 2009, Orlando, Florida

23. Mahnken AH, Raupach R, Wildberger JE, et al. A new algorithm for metal artifact reduction in computed tomography: in vitro and in vivo evaluation after total hip replacement. Invest Radiol 2003;38:769-75

24. Fahrig R, Dixon R, Payne T, et al. Dose and image quality for a cone-beam C-arm CT system. Med Phys 2006;33:4541-50

25. Prell D, Kyriakou Y, Kalender WA. Comparison of ring artifact correction methods for flat-detector CT. Phys Med Biol 2009;54:3881-95

26. Schiwietz T, Bose S, Maltz J, et al. A fast and high-quality cone beam reconstruction pipeline using the GPU. Med Imaging 2007; Physics of Medical Imaging, Proc. SPIE, vol. 6516, 16; March 2007; Lake Buena Vista, Florida

27. Kyriakou Y, Lapp RM, Hillebrand L, et al. Simultaneous misalignment correction for approximate circular cone-beam computed tomography. Phys Med Biol 2008;53:267-89 